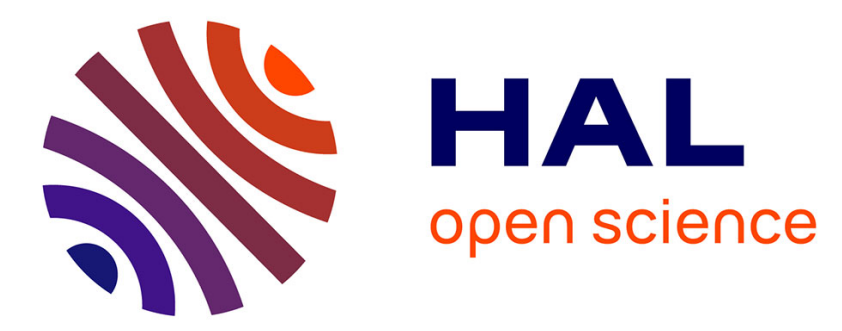

\title{
Discontinuities, generalized solutions and (dis)agreement in opinion dynamics
}

Francesca Ceragioli, Paolo Frasca

\section{To cite this version:}

Francesca Ceragioli, Paolo Frasca. Discontinuities, generalized solutions and (dis)agreement in opinion dynamics. Sophie Tarbouriech; Antoine Girard; Laurentiu Hetel. Control subject to computational and communication constraints, 475, Springer, pp.287-309, 2018, Lecture Notes in Control and Information Sciences, 978-3-319-78449-6. 10.1007/978-3-319-78449-6_14 . hal-01739420

\section{HAL Id: hal-01739420 \\ https://hal.science/hal-01739420}

Submitted on 21 Mar 2018

HAL is a multi-disciplinary open access archive for the deposit and dissemination of scientific research documents, whether they are published or not. The documents may come from teaching and research institutions in France or abroad, or from public or private research centers.
L'archive ouverte pluridisciplinaire HAL, est destinée au dépôt et à la diffusion de documents scientifiques de niveau recherche, publiés ou non, émanant des établissements d'enseignement et de recherche français ou étrangers, des laboratoires publics ou privés. 


\title{
Discontinuities, generalized solutions and (dis)agreement in opinion dynamics
}

\author{
Francesca Ceragioli and Paolo Frasca
}

\begin{abstract}
This chapter is devoted to the mathematical analysis of some continuoustime dynamical systems defined by ordinary differential equations with discontinuous right-hand side, which arise as models of opinion dynamics in social networks. Discontinuities originate because of specific communication constraints, namely quantization or bounded confidence. Solutions of these systems may or may not converge to a state of agreement, where all components of the state space are equal. After presenting three models of interest, we elaborate on the properties of their solutions in terms of existence, completeness, and convergence.
\end{abstract}

\section{Discontinuous consensus-seeking systems}

This chapter studies some continuous-time dynamical systems defined by ordinary differential equations with discontinuous right-hand side. The dynamics under consideration have been proposed in the last fifteen years in the context of "consensusseeking" systems, which describe coordination phenomena in engineering, biology and social sciences. Given this range of applications, the reader will not be surprised that we are dealing with rather abstract representations of reality.

The most basic consensus-seeking system takes the following form. Let $x$ be an $N$-dimensional vector, where each component $x_{i}$ is associated to an individual $i \in$ $\mathscr{I}=\{1, \ldots, N\}$ and evolves in time according to the ordinary differential equation

Francesca Ceragioli

Politecnico di Torino, c.so Duca degli Abruzzi 24, 10129 Torino, Italy e-mail: francesca.ceragioli@polito.it

Paolo Frasca

Univ. Grenoble Alpes, CNRS, Inria, Grenoble INP, GIPSA- Lab, 38000 Grenoble, France. e-mail: paolo.frasca@gipsa-lab.fr 


$$
\dot{x}_{i}(t)=\sum_{j=1}^{N} a_{i j}\left(x_{j}(t)-x_{i}(t)\right) \quad i \in \mathscr{I} .
$$

Since we assume that the interaction weights $a_{i j}$ are nonnegative, this dynamics postulates that each individual is attracted by the other individuals with whom it interacts. Under very mild assumptions on the interaction pattern, this dynamics converges to a state of agreement where all components $x_{i}$ are equal.

Variations to this dynamics have been proposed in order to accommodate a host of phenomena, including time- and state-dependent interactions $a_{i j}(t, x)$. An interesting case of state-dependent interactions is the following, which is termed bounded confidence in the literature. Two individuals are assumed to influence each other if their states are closer than a certain threshold (that we choose to be 1 for simplicity):

$$
\begin{aligned}
& \dot{x}_{i}(t)=\sum_{j=1}^{N} a\left(x_{i}(t), x_{j}(t)\right)\left(x_{j}(t)-x_{i}(t)\right) \quad i \in \mathscr{I} \\
& \text { where } a(y, z)= \begin{cases}1 & \text { if }|y-z|<1 \\
0 & \text { if }|y-z| \geq 1 .\end{cases}
\end{aligned}
$$

This model, which is a continuous-time counterpart of the opinion dynamics studied by Hegselmann \& Krause [34], has been proposed by [8] and further considered in [15]. Very similar models have been considered in [40, 35, 51, 19, 49]. We will see that (BC) does not produce agreement, but clustering of individuals into groups characterised by agreement within each group and disagreement between groups.

Another relevant phenomenon is quantization, which occurs both in engineering and in social systems. In engineering, it can represent communication constraints, where the state variable is communicated between individuals via a digital channel with finite data rate, and thus constrained to take on discrete values. For the sake of this analysis, we shall define the quantization of a real number simply by rounding it to the closest integer: $q(s)=\left\lfloor s+\frac{1}{2}\right\rfloor$. In this context, an effective consensus-seeking system is the following "quantized states" system studied in [14]:

$$
\dot{x}_{i}(t)=\sum_{j=1}^{N} a_{i j}\left(q\left(x_{j}(t)\right)-q\left(x_{i}(t)\right)\right) \quad i \in \mathscr{I} .
$$

Note that the right-hand side features the quantized values of both states $x_{j}$ and $x_{i}$ : the presence of the quantized state $q\left(x_{i}\right)$ is crucial to ensure the "good" properties of this dynamics, which will be discussed below.

In social systems, quantization may originate because the state variable is "communicated" as the display of an action or behavior, which can take on discrete values only: for instance, the purchase of certain products. In this context, we have recently proposed $[16,17]$ to investigate the following "quantized behaviors" model: 


$$
\dot{x}_{i}(t)=\sum_{j=1}^{N} a_{i j}\left[q\left(x_{j}(t)\right)-x_{i}(t)\right] \quad i \in \mathscr{I}=\{1, \ldots, N\}
$$

Note that, in contrast with (QS), the right-hand side features the quantized value of $x_{j}$, but not of $x_{i}$, which leads to more complex dynamics than (QS).

The reader can notice that the right-hand sides of equations $(B C),(Q S),(Q B)$ are discontinuous in the state variable. Since the study of these non-smooth systems relies on relatively sophisticated instruments that might not always be accessible to the non-specialists, we have included in this chapter an extended review of the necessary mathematical machinery, which we hope can be of independent interest. Nevertheless, readers are advised to consult the literature specific to the topic, for instance the tutorial [21] and the books [4, 24], as well as more specific works about stability [6] or generalized solutions [12]. Additional references are provided in the following sections.

The rest of this paper is organized as follows. Section 2 defines some useful notation and summarizes well-known results from graph theory together with their consequences for the consensus dynamics (1). Section 3 presents some notions of solutions that are relevant in this context, namely those of Carathéodory and Krasovskii. The section also contains results on existence and completeness of these solutions for a general class of piece-wise affine systems and specifically for the three dynamics at hand. Section 4 deals with equilibria (again, declined according to the relevant notions of solutions) and describes the sets of equilibria for our three dynamics. Section 5 deals with convergence of their trajectories. We provide two kinds of results: on the one hand, sufficient conditions to reach agreement, and on the other hand, general statements about convergence to the equilibria (or to their proximity).

Owing to the survey purpose that we set us for this paper, we have avoided reporting the details of some proofs that can be easily found in the literature. Furthermore, we restrict our presentation to assume symmetric interactions, namely such that $a_{i j}=a_{j i}$ for all $i, j$ in $\mathscr{I}$. We made this choice for simplicity of exposition, even though most results can be extended to non-symmetric interactions.

\section{Preliminaries}

Notation. Given a subset $S$ of $\mathbb{R}^{N}$, we denote by $\bar{S}$ its topological closure, by $\partial S$ its border, and by $\overline{c o} S$ its closed convex hull. We let $\mathbf{0}=(0, \ldots, 0)^{\top}, \mathbf{1}=(1, \ldots, 1)^{\top}$ and $e_{i}, i=\{1, \ldots, N\}$, the vectors of the canonical basis of $\mathbb{R}^{N}$. We call consensus point a point of the form $\alpha \mathbf{1}$ with $\alpha \in \mathbb{R}$. The $N$-dimensional identity matrix is denoted by $I$ and $\|\cdot\|$ denotes the Euclidean norm both for vectors and matrices. Given the vector $x \in \mathbb{R}^{N}$, we denote its average by $x_{\text {ave }}=\frac{1}{N} \mathbf{1}^{\top} x=\frac{1}{N} \sum_{i=1}^{N} x_{i}$. When $x=x(t)$ we shall write $x_{\text {ave }}(t)=\frac{1}{N} \sum_{i=1}^{N} x_{i}(t)$. The notation $q(x)$ with $x \in \mathbb{R}^{N}$ will denote the vector whose $i$-th componenet is $q\left(x_{i}\right)$. 
Graph theory. A weighted (undirected) graph $\mathscr{G}=(\mathscr{I}, \mathscr{E}, A)$ consists of a node set $\mathscr{I}=\{1, \ldots, N\}$, an edge set $\mathscr{E} \subset \mathscr{I} \times \mathscr{I}$, and a symmetric adjacency matrix $A \in \mathbb{R}_{+}^{N \times N}$ such that $a_{i j}>0$ if $(i, j) \in \mathscr{E}$, and $a_{i j}=0$ if $(j, i) \notin \mathscr{E}$. We assume no self-loops in the graph, that is $a_{i i}=0$ for all $i \in \mathscr{I}$. Nodes (vertices) are referred to as agents or individuals, edges as links. Let $d_{i}:=\sum_{j=1}^{N} a_{i j}$ be the degree of node $i \in \mathscr{I}$. Let $D=\operatorname{diag}(A \mathbf{1})$ be the diagonal matrix whose diagonal entries are the degrees of each node. Let $L=D-A$ be the Laplacian matrix of the graph $\mathscr{G}$. Note that by construction $L \mathbf{1}=\mathbf{0}$ and by symmetry $\mathbf{1}^{\top} L=\mathbf{0}^{\top}$. In case the graph is state dependent, we write $\mathscr{G}(x), \mathscr{E}(x), A(x), D(x), L(x)$.

Given an edge $(i, j)$, we shall refer to $i$ and to $j$ as the tail and the head of the edge, respectively. A path is an ordered list of edges such that the head of each edge is equal to the tail of the following one. The graph $\mathscr{G}$ is said to be connected if for any $i, j \in \mathscr{I}$ there is a path from $i$ to $j$ in $\mathscr{G}$. If the graph is connected, then the eigenvalue 0 of the Laplacian matrix $L$ has algebraic multiplicity 1 . The vector $x-x_{\text {ave }} \mathbf{1}$ is the projection of $x$ on the subspace orthogonal to $\mathbf{1}$ : consequently, if we denote by $\lambda_{*}$ the smallest non-zero eigenvalue of $L$, one has

$$
\left(x-x_{\text {ave }} \mathbf{1}\right)^{\top} L\left(x-x_{\text {ave }} \mathbf{1}\right) \geq \lambda_{*}\left\|x-x_{\text {ave }} \mathbf{1}\right\|^{2} \forall x \in \mathbb{R}^{N} .
$$

Convergence to agreement. Using the Laplacian matrix, dynamics (1) can be compactly rewritten as

$$
\dot{x}=-L x
$$

Its key properties, descending from the properties of the Laplacian that we recalled above, are summarized by the following well-known result and illustrated in Figure 1 .

Theorem 1 (Real consensus). If the graph underlying (1) is connected and the adjacency matrix $A$ is symmetric, then for any solution $x(t)$ of (1) the following properties hold true:

1. (contractivity and boundedness) $\overline{c o}\left\{x_{i}(t), i \in \mathscr{I}\right\} \subseteq \overline{c o}\left\{x_{i}(0), i \in \mathscr{I}\right\}$;

2. (average preservation) $x_{\text {ave }}(0)=x_{\text {ave }}(t)$;

3. (equilibria) $x^{*}$ is an equilibrium point of (1) if and only if $x^{*}$ is a consensus point;

4. (average consensus) $\lim _{t \rightarrow+\infty} x(t)=x_{\text {ave }}(0) \mathbf{1}$.

\section{Generalized solutions and basic properties of the dynamics}

In this section we summarize some notions which are essential in order to deal with systems whose right-hand side is discontinuous with respect to the state variable.

Let us consider the Cauchy problem

$$
\dot{x}=f(x) \quad x(0)=x_{0}
$$




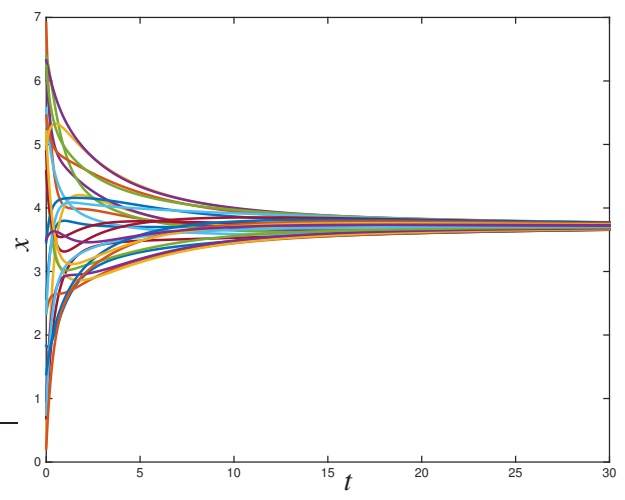

Fig. 1 Evolution of a solution of (1) from random initial conditions on a cycle graph on 25 nodes.

where $x_{0} \in \mathbb{R}^{N}$ and $f: \mathbb{R}^{N} \rightarrow \mathbb{R}^{N}$ is measurable and locally bounded. We will denote by $\Delta_{f}$ the subset of $\mathbb{R}^{N}$ where $f$ is discontinuous. When facing system (3), one should first of all choose which type of generalized solution is the most suitable for the system of interest. We shall consider Carathéodory solutions and Krasovskii solutions.

\subsection{Carathéodory solutions}

The notion of solution nearest to the classical one is that of Carathéodory solution.

Definition 1 (Carathéodory solution). Let $I \subset \mathbb{R}$ be an interval with $0 \in I$ and let $x_{0} \in \mathbb{R}^{N}$. An absolutely continuous function $\varphi: I \rightarrow \mathbb{R}^{N}$ is a Carathéodory solution of equation (3) on $I$ with initial condition $x_{0}$ if $\varphi(0)=x_{0}$ and if it satisfies (3) for almost all $t \in I$ or, equivalently, if it is a solution of the integral equation

$$
\varphi(t)=x_{0}+\int_{0}^{t} f(\varphi(s)) d s
$$

We say that a local Carathéodory solution corresponding to the initial condition $x_{0} \in \mathbb{R}$ exists if there there exist a neighbourhood $I\left(x_{0}\right)$ of $x_{0}$, an interval of the form $[0, T)$ and an absolutely continuous function $\varphi:[0, T) \rightarrow I\left(x_{0}\right)$ such that $\varphi(0)=x_{0}$ and $\varphi(t)$ is a Carathéodory solution of (3) on $[0, T)$.

Note that in the models we are considering the set $\Delta_{f}$ of discontinuity points of the vector field $f(x)$ has a particularly simple structure, as it can be locally represented as the union of a finite number of hyperplanes. This observation is made rigorous by the following assumption.

Assumption 1 (On the discontinuity set) For any $x_{0} \in \Delta_{f}$ there exists a neighourhood $I\left(x_{0}\right)$ of $x_{0}$ and $m$ affine functions $s_{1}, \ldots, s_{m}: I\left(x_{0}\right) \rightarrow \mathbb{R}^{N}$ defined by 


$$
s_{\ell}(x)=p_{\ell}^{\top} x-c_{\ell} \quad \ell \in\{1, \ldots, m\}
$$

with $p_{\ell} \in \mathbb{R}^{N}$ and $c_{\ell} \in \mathbb{R}$, such that $s_{\ell}\left(x_{0}\right)=0$ for all $\ell \in\{1, \ldots, m\}$ and

$$
\Delta_{f} \cap I\left(x_{0}\right)=\left\{x \in I\left(x_{0}\right): s_{1}(x)=0\right\} \cup \ldots \cup\left\{x \in I\left(x_{0}\right): s_{m}(x)=0\right\} .
$$

Under Assumption 1, the neighbourhood $I\left(x_{0}\right)$ of $x_{0}$ is partitioned in $2^{m}$ sectors $S_{\mathbf{b}}\left(x_{0}\right)$ defined by the signs of the functions $s_{1}, \ldots, s_{m}$, indexed by means of $\mathbf{b} \in$ $\{-1,1\}^{m}$, and defined in the following way ${ }^{1}$ :

$S_{\mathbf{b}}\left(x_{0}\right)=\left\{x \in I\left(x_{0}\right): s_{\ell}(x)<0\right.$ if $b_{\ell}=-1$ and $s_{\ell}(x) \geq 0$ if $\left.b_{\ell}=1, \ell=1, \ldots, m\right\}$.

Assumption 2 (On the discontinuous vector field) The parts $S_{\mathbf{b}}\left(x_{0}\right)$ are defined so that the vector field $f(x)$ is continuous on $S_{\mathbf{b}}\left(x_{0}\right)$ for all $\mathbf{b} \in\{-1,1\}^{m}$.

Without Assumption 2, the choice of the representation of the discontinuity hyperplanes by means of $p_{\ell}$ and $c_{\ell}$ would not be unique, since the orientation of the normal vector is arbitrary. Assumption 2 makes sure that the choice of the representation is consistent with the functions $a(\cdot, \cdot)$ and $q(\cdot)$ in equations (BC), (QS), (QB).

Under Assumptions 1 and 2, the vector field $f(x)$ has $2^{m}$ limit values as $x \rightarrow x_{0}$, namely

$$
f^{\mathbf{b}}\left(x_{0}\right)=\lim _{x \in S_{\mathbf{b}}, x \rightarrow x_{0}} f(x) .
$$

Example 1 (BC dynamics with 3 individuals). Consider dynamics (BC) with $N=3$ and $x_{0}=(0,1,2)^{\top}$. Clearly, point $x_{0}$ lies at the intersection of the two planes of discontinuity $x_{2}-x_{1}-1=0$ and $x_{3}-x_{2}-1=0$, namely defined by the normal vectors $p_{1}=(-1,1,0)^{\top}$ and $p_{2}=(0,-1,1)^{\top}$. In the sectors $S_{(1,1)}, S_{(-1,1)}, S_{(1,-1)}, S_{(-1,-1)}$, we respectively identify the four limit values of the vector field

$$
\begin{array}{ll}
f^{(1,1)}\left(x_{0}\right)=\left(\begin{array}{l}
0 \\
0 \\
0
\end{array}\right) \quad f^{(-1,1)}\left(x_{0}\right)=\left(\begin{array}{c}
1 \\
-1 \\
0
\end{array}\right) \\
f^{(1,-1)}\left(x_{0}\right)=\left(\begin{array}{c}
0 \\
1 \\
-1
\end{array}\right) & f^{(-1,-1)}\left(x_{0}\right)=\left(\begin{array}{c}
1 \\
0 \\
-1
\end{array}\right) .
\end{array}
$$

This situation is represented in Figure 2.

Under Assumptions 1 and 2, the study of existence and completeness of Carathéodory solutions can be relatively simple. Nevertheless one can not expect to have local existence in general: the negative example is given by the following proposition $[33,13]$, illustrated in the fourth diagram of Figure 3.

\footnotetext{
${ }^{1}$ It would be more precise to write $S_{\mathbf{b}}\left(x_{0}, I\left(x_{0}\right)\right)$ instead of $S_{\mathbf{b}}\left(x_{0}\right)$, as it depends on $I\left(x_{0}\right)$. Note however that if $I\left(x_{0}\right)$ and $I^{\prime}\left(x_{0}\right)$ are two distinct neighbourhoods of $x_{0}$, then the sets $S_{\mathbf{b}}\left(x_{0}, I\left(x_{0}\right)\right)$ and $S_{\mathbf{b}}\left(x_{0}, I^{\prime}\left(x_{0}\right)\right)$ coincide on $I\left(x_{0}\right) \cap I^{\prime}\left(x_{0}\right)$. Hence neglecting $I\left(x_{0}\right)$ from the notation brings no ambiguity.
} 


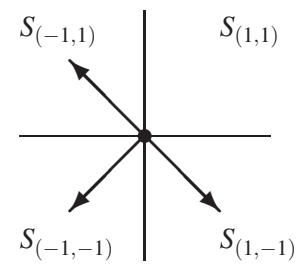

Fig. 2 Representation of the limit values of the vector field at a discontinuity point where two hyperplanes intersect, namely dynamics $(\mathrm{BC})$ at point $(0,1,2)^{\top}$.

Proposition 1 (Non-existence of Carathéodory solutions). Let $x_{0} \in \Delta_{f}$ and assume $m=1$ in Assumption 1. If $p_{1}^{\top} f^{(-1)}\left(x_{0}\right)>0$ and $p_{1}^{\top} f^{(1)}\left(x_{0}\right)<0$, then there exists no Carathéodory solution of (3) with initial condition $x_{0}$.

An example of this situation is given by dynamics (QS).

Example 2 (Non-existence in QS dynamics). Consider dynamics (QS) over an undirected path graph with $N=3$ whose adjacency matrix $A$ has all non-null entries equal to 1 and the initial condition $\bar{x}_{0}=(1,3 / 2,2)^{\top}$. The right-hand side of the system is clearly discontinuous at $\bar{x}_{0}$. There exists a neighbourhood $I\left(\bar{x}_{0}\right)$ of $\bar{x}_{0}$ such that $\Delta_{f} \cap I\left(\bar{x}_{0}\right)=\left\{x \in \mathbb{R}^{N}: x_{2}-3 / 2=0\right\}$ and we thus define $s_{1}(x)=x_{2}-3 / 2=$ $(0,1,0) x-3 / 2$. We get that $f^{(-1)}\left(\bar{x}_{0}\right)=(0,1,-1)^{\top}$ and $f^{(1)}\left(\bar{x}_{0}\right)=(1,-1,0)^{\top}$, then $(0,1,0) f^{(1)}\left(\bar{x}_{0}\right)=-1<0$ and $(0,1,0) f^{(-1)}\left(\bar{x}_{0}\right)>0$. By applying Proposition 1 we conclude that there are no Carathéodory solutions issuing from $\bar{x}_{0}$.
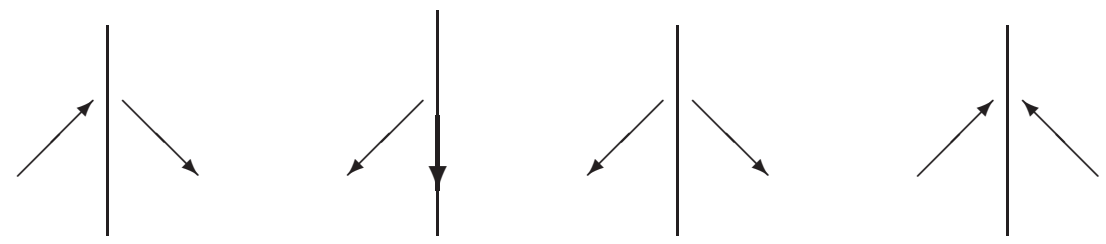

Fig. 3 Representations of the possible orientations of the vector fields in the neighborhood of a discontinuity hyperplane.

Instead, the following result provides a sufficient condition for the existence of local Carathéodory solutions. It is inspired by the concept of directional continuity in [44] but it allows also for solutions lying on the discontinuity set: this case, which can be particularly subtle to be treated, is simplified here by the discontinuity being a union of hyperplanes. Informally, the sufficient condition requires that, for each discontinuity point, at least one among the "pieces" of the vector field either pulls 
away from the discontinuity surface in its own sector, or is parallel to a discontinuity hyperplane.

Theorem 2 (Sufficient condition for Carathéodory solutions). Assume that Assumptions 1 and 2 hold. Assume that for any $x_{0} \in \Delta_{f}$ there exists $\tilde{\mathbf{b}} \in\{-1,1\}^{m}$ such that

1. $\left[p_{\ell}^{\top} f^{\tilde{\mathbf{b}}}\left(x_{0}\right)\right] \tilde{b}_{\ell} \geq 0$ for all $\ell \in\{1, \ldots, m\}$;

2. if there exists $\bar{\ell} \in\{1, \ldots, m\}$ such that $\left[p_{\bar{\ell}}^{\top} f^{\tilde{\mathbf{b}}}\left(x_{0}\right)\right] \tilde{b}_{\bar{\ell}}=0$, then there exists a neighborhood $J\left(x_{0}\right)$ such that, for all $x \in J\left(x_{0}\right) \cap \partial S_{\tilde{\mathbf{b}}}$, both $\left[p_{\bar{\ell}}^{\top} f(x)\right] \tilde{b}_{\bar{\ell}}=0$ and the restriction $\left.f\right|_{\left(J\left(x_{0}\right) \cap \partial S_{\tilde{\mathbf{b}}}\right) \backslash\left\{x_{0}\right\}}$ is continuous.

Then, there exists a local Carathéodory solution issuing from $x_{0}$.

Proof. If $p_{\ell}^{\top} f^{\tilde{\mathbf{b}}}\left(x_{0}\right) \tilde{b}_{\ell}>0$ for all $\ell \in\{1, \ldots, m\}$, then the vector field points into the interior of $S_{\tilde{\mathbf{b}}}$ and a local solution can be easily constructed as in [44]. In the case of condition 2 , i.e. when the vector field $f(x)$ is parallel to one of the discontinuity hyperplanes in a neighbourhood of $x_{0}$ except, possibly, in $x_{0}$, one can still construct a sequence of Euler polygonal chains that lie on the hyperplane and converge to a Carathéodory solution.

Condition 2 in Theorem 2 is a relaxation of [13, Assumption (H3)], which is made possible by the fact that discontinuities are (locally) hyperplanes. A simple application of Theorem 2 is the following.

Example 3 (BC dynamics with 3 individuals-Continued). Consider again dynamics (BC) with $N=3$ and $x_{0}=(0,1,2)^{\top}$, as illustrated in Figure 2. We observe that in the four sectors around $x_{0}$, $p_{1}^{\top} f^{(1,1)}\left(x_{0}\right)(1)=0$ and $p_{2}^{\top} f^{(1,1)}\left(x_{0}\right)(1)=0$ $p_{1}^{\top} f^{(-1,1)}\left(x_{0}\right)(-1)=(-2)(-1)>0$ and $p_{2}^{\top} f^{(-1,1)}\left(x_{0}\right)(1)=(1)(1)>0$ $p_{1}^{\top} f^{(1,-1)}\left(x_{0}\right)(1)=(1)(1)>0$ and $p_{2}^{\top} f^{(1,-1)}\left(x_{0}\right)(-1)=(-2)(-1)>0$ $p_{1}^{\top} f^{(-1,-1)}\left(x_{0}\right)(-1)=(-1)(-1)>0$ and $p_{2}^{\top} f^{(-1,-1)}\left(x_{0}\right)(-1)=(-1)(-1)>0$. Then, Theorem 2 implies local existence. Actually, one can see that in this case four solutions originate from $x_{0}$, one for each sector: they are shown in Figure 4 as functions of time.

More in general, we can prove existence of Carathéodory solutions of (BC) for any initial condition. Carathéodory solutions of (BC) were studied in [7] where existence and uniqueness of solutions were proved for almost all initial condition. Here we prove ${ }^{2}$ existence for all initial conditions and we remark that in general we do not have uniqueness. The proof is a verification of the assumptions of Theorem 2 , where we see that the sole strictly positive case suffices.

Corollary 1 (Existence for BC). For any initial condition there exists a local Carathéodory solution of (BC).

${ }^{2}$ Even though the corollary is new, it could have been deduced by inspecting the proofs in [7]. 

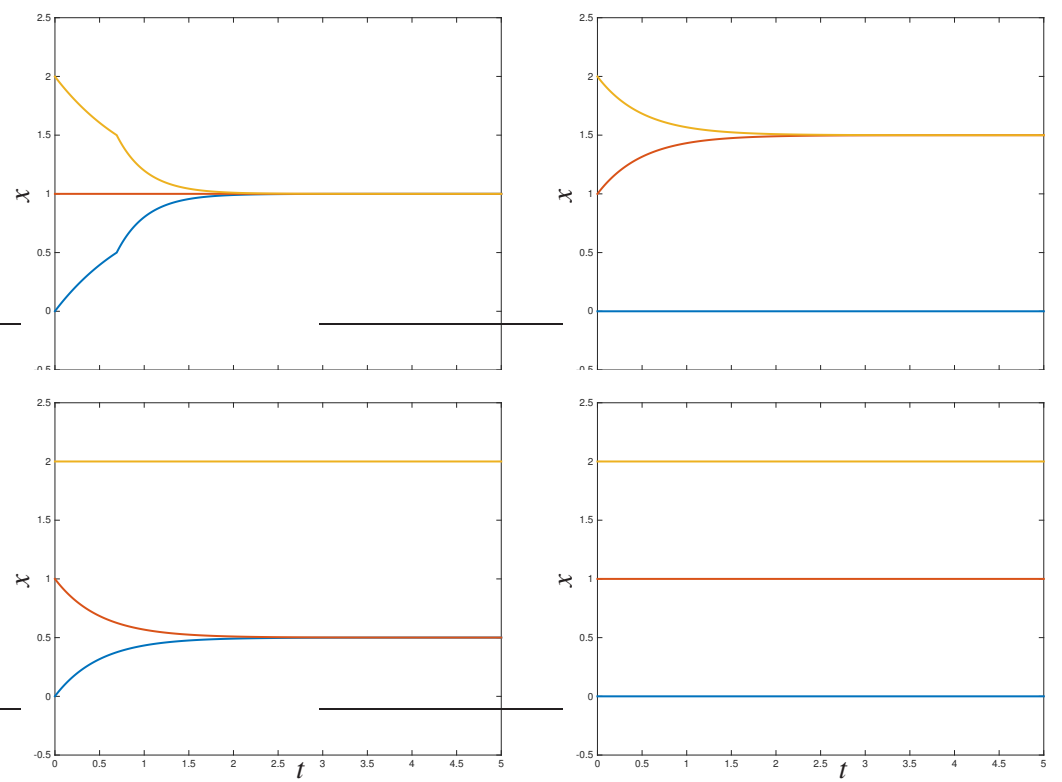

Fig. 4 Evolutions of the four solutions of (BC) that originate from $x_{0}=(0,1,2)^{\top}$.

Proof. We denote by $f(x)$ the right-hand side of (BC) and observe that $\Delta_{f}=\{x \in$ $\mathbb{R}: \exists i, j \in \mathscr{I}$ such that $\left.x_{i}-x_{j}=1\right\}$. We first consider $x_{0} \in \Delta_{f}$ in the case $x_{0 i}-x_{0 j}=1$ for only one pair of indices $i, j \in \mathscr{I}$. In this case $m=1$ in Assumption $1, s_{1}(x)=$ $x_{i}-x_{j}-1=\left(e_{i}-e_{j}\right)^{\top} x-1$, and as $b$ is either -1 or 1 ,

$S_{(-1)}\left(x_{0}\right)=\left\{x \in \mathbb{R}^{N}: x_{i}-x_{j}-1<0\right\}$,

$\left(f^{(-1)}\left(x_{0}\right)\right)_{i}=\sum_{h \neq j:\left|x_{0 h}-x_{0 i}\right|<1}\left(x_{h}-x_{i}\right)-1$,

$\left(f^{(-1)}\left(x_{0}\right)\right)_{j}=\sum_{h \neq i:\left|x_{0 i}-x_{0 j}\right|<1}\left(x_{h}-x_{j}\right)+1$,

as well as

$S_{(1)}\left(x_{0}\right)=\left\{x \in \mathbb{R}^{N}: x_{i}-x_{j}-1 \geq 0\right\}$,

$\left(f^{(1)}\left(x_{0}\right)\right)_{i}=\sum_{h \neq j:\left|x_{0 i}-x_{0 i}\right|<1}\left(x_{h}-x_{i}\right)$,

$\left(f^{(1)}\left(x_{0}\right)\right)_{j}=\sum_{h \neq i:\left|x_{0 i}-x_{0 j}\right|<1}\left(x_{h}-x_{j}\right)$.

We then get $\left(e_{i}-e_{j}\right)^{\top} f^{(-1)}\left(x_{0}\right)=\left[\sum_{h \neq j:\left|x_{h}-x_{i}\right|<1}\left(x_{h}-x_{i}\right)-\sum_{h \neq i:\left|x_{h}-x_{i}\right|<1}\left(x_{h}-x_{i}\right)\right]-$ 2 and $\left(e_{i}-e_{j}\right)^{\top} f^{(1)}\left(x_{0}\right)=\left[\sum_{h \neq j:\left|x_{h}-x_{i}\right|<1}\left(x_{h}-x_{i}\right)-\sum_{h \neq i:\left|x_{h}-x_{i}\right|<1}\left(x_{h}-x_{i}\right)\right]$.

If $\left[\sum_{h \neq j:\left|x_{h}-x_{i}\right|<1}\left(x_{h}-x_{i}\right)-\sum_{h \neq i:\left|x_{h}-x_{i}\right|<1}\left(x_{h}-x_{i}\right)\right]>0$, then $\left[\left(e_{i}-e_{j}\right)^{\top} f^{(1)}\left(x_{0}\right)\right](1)>$ 0 and condition 1 of Theorem 2 is verified and a Carathéodory solution entering $S_{(1)}$ exists. If $\left[\sum_{h \neq j:\left|x_{h}-x_{i}\right|<1}\left(x_{h}-x_{i}\right)-\sum_{h \neq i:\left|x_{h}-x_{i}\right|<1}\left(x_{h}-x_{i}\right)\right] \leq 0$ then $\left[\left(e_{i}-\right.\right.$ $\left.\left.e_{j}\right)^{\top} f^{(-1)}\left(x_{0}\right)\right]=\left[\left(e_{i}-e_{j}\right)^{\top} f^{(1)}\left(x_{0}\right)\right]-2<0$ and $\left[\left(e_{i}-e_{j}\right)^{\top} f^{(-1)}\left(x_{0}\right)\right](-1)>0$, so that a Carathéodory solution issuing from $x_{0}$ and entering $S_{(-1)}$ exists.

In case $m>1$ in Assumption 1, i.e. $x_{0 i}-x_{0 j}=1$ for more than one pair $(i, j)$, one starts by considering the set $S_{(1,1, \ldots, 1)}$ and the vector $f^{(1,1, \ldots, 1)}\left(x_{0}\right)$. If $\left[p_{\ell}^{\top} f^{(1,1, \ldots, 1)}\left(x_{0}\right)\right](1)>0$ for all $\ell \in\{1, \ldots, m\}$ then condition 1 of Theorem 2 is ver- 
ified. Otherwise there exists $\bar{\ell} \in\{1, \ldots, m\}$ such that $\left[p_{\bar{\ell}}^{\top} f^{(1,1, \ldots, 1)}\left(x_{0}\right)\right](1) \leq 0$. In this case we consider the set $S_{(1, \ldots,-1, \ldots, 1)}$ and the vector $f^{(1, \ldots,-1, \ldots, 1)}\left(x_{0}\right)$. From the previous step we know that for $\ell=1, \ldots, \bar{\ell}-1$ one has $\left[p_{\ell}^{\top} f^{(1, \ldots,-1, \ldots, 1)}\left(x_{0}\right)\right](1)>0$ and $\left[p_{\bar{\ell}}^{\top} f^{(1, \ldots,-1, \ldots, 1)}\left(x_{0}\right)\right](-1)=\left[p_{\bar{\ell}}^{\top} f^{(1,1, \ldots, 1)}\left(x_{0}\right)-2\right](-1)>0$. Now, if for $\ell=$ $\bar{\ell}+1, \ldots, m$ one has $\left[p_{\ell}^{\top} f^{(1, \ldots,-1, \ldots, 1)}\left(x_{0}\right)\right](1)>0$, then condition 1 is satisfied, otherwise one goes on with the same procedure. We remark that if $\left[p_{\bar{\ell}}^{\top} f^{(1,1, \ldots 1)}\left(x_{0}\right)\right] \leq 0$, then $\left[p_{\bar{\ell}}^{\top} f^{\mathbf{b}}\left(x_{0}\right)\right] \leq 0$ for all $\mathbf{b} \in\{-1,1\}^{m}$. The procedure stops after at most $m$ steps, having checked all the sectors, returning a certificate for condition 1 .

Regarding (QB), the following corollary of Theorem 2 is proved in [17]: in this case, condition 2 of Theorem 2 needs to be applied.

Corollary 2 (Existence for QB). For any initial condition there exists a local Carathéodory solution of $(\mathrm{QB})$.

We emphasize that Carathéodory solutions may not be unique, as shown in Example 3. Another example of non-uniqueness is given by $(\mathrm{QB})$.

Example 4. Consider the discrete behaviour dynamics $(\mathrm{QB})$ over the undirected path graph with $N=2$ whose adjacency matrix $A$ has non-null entries equal to 1 and the initial condition $\bar{x}_{0}=(1 / 2,1 / 2)^{\top}$. The right-hand side of the system is clearly discontinuous at $\bar{x}_{0}$. There are two solutions issuing from this point which correspond to the limit values of $f(x)$ when restricted to the two sets $S_{(-1,1)}=\left\{x \in \mathbb{R}^{2}: x_{i}-\frac{1}{2}<\right.$ $0, i=1,2\}$ and $S_{(1,1)}=\left\{x \in \mathbb{R}^{2}: x_{i}-\frac{1}{2} \geq 0, i=1,2\right\}$. These solutions converge to $(0,0)^{\top}$ and $(1,1)^{\top}$, respectively. Their trajectories are the line segments joining the initial condition with the points $(0,0)^{\top}$ and $(1,1)^{\top}$; see Figure 5 .

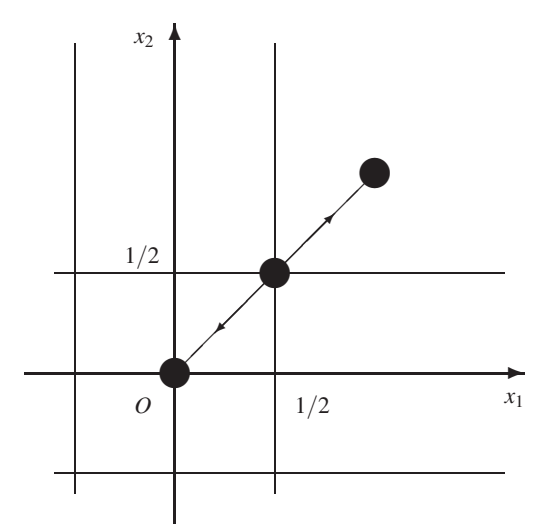

Fig. 5 Lack of unicity in dimension 2 for dynamics (QB), see Example 4. 


\subsection{Krasovskii solutions}

In order to cope with non-existence of solutions, other generalized solutions have been introduced in the literature. In the context described here, Krasovskii solutions can be easily and successfully used.

Definition 2 (Krasovskii solutions). Let $I \subset \mathbb{R}$ be an interval with $0 \in I$ and let $x_{0} \in \mathbb{R}^{N}$. An absolutely continuous function $\varphi: I \rightarrow \mathbb{R}^{N}$ is a Krasovskii solution of (3) with initial condition $x_{0}$ if $\varphi(0)=x_{0}$ and if for almost all $t \in I$ it satisfies the differential inclusion

$$
\dot{\varphi}(t) \in \mathscr{K} f(\varphi(t)),
$$

where

$$
\mathscr{K} f(x)=\bigcap_{\delta>0} \overline{c o}\{f(y): y \text { such that }\|x-y\|<\delta\} .
$$

We say that a local Krasovskii solution corresponding to the initial condition $x_{0} \in$ $\mathbb{R}^{N}$ exists if there exist a neighbourhood $I\left(x_{0}\right)$ of $x_{0}$, an interval of the form $[0, T)$ and an absolutely continuous function $\varphi:[0, T) \rightarrow I\left(x_{0}\right)$ such that such that $\varphi(0)=x_{0}$ and $\varphi(t)$ is a Krasovskii solution of (3) on $[0, T)$.

The following existence theorem is an immediate consequence of [4, Theorem 3 , page 98], as the vector field $f(x)$ is measurable and locally bounded.

Theorem 3. For any initial condition $x_{0} \in \mathbb{R}^{N}$ there exists a local Krasovskii solution of (3).

We underline that any Carathéodory solution is also a Krasovskii solution. Another type of generalized solutions often adopted for discontinuous systems are Filippov solutions ([24]). Under Assumptions 1 and 2, Krasovskii solutions coincide with Filippov solutions (see [33]) .

\subsection{Completeness of solutions}

Besides local existence, we are interested in solutions that are defined on unbounded intervals. The following condition is well-known: its proof is included for completeness and tutorial purposes.

Proposition 2 (Prolongation by boundedness). Let (3) be a system that admits local Carathéodory (Krasovskii) solutions for every initial condition in $\mathbb{R}^{N}$. Let $\varphi$ : $[0, T) \rightarrow \mathbb{R}^{N}$ be a Carathéodory (Krasovskii) solution of (3). If $\varphi(t)$ is bounded on $[0, T)$, then it can be continued over $\left[0, T^{\prime}\right)$ with $T^{\prime}>T$.

Proof. Assume by contradiction that there exists an initial condition $x_{0}$ whose corresponding maximal Carathéodory (Krasovskii) right solution has domain $[0, T)$ with $T<+\infty$ and let $\left\{t_{n}\right\}$ be a sequence such that $t_{n}>0$ and $\lim t_{n}=T$. Since $x(t)$ is 
bounded also the sequence $\left\{x\left(t_{n}\right)\right\}$ is bounded and thus there exists a subsequence $x\left(t_{n_{k}}\right)$ converging to a point $x^{*}$. Since $x(t)$ is continuous this implies that there exists $\lim _{t \rightarrow T} x(t)=x^{*} \in \mathbb{R}^{N}$. One can then pose a new Cauchy problem with initial condition $x(T)=x^{*}$ and then continue the Carathéodory (Krasovskii) solution on an interval $\left[T, T^{\prime}\right)$. We then get to a contradiction as the interval $[0, T)$ is not maximal for the considered Carathéodory (Krasovskii) solution with initial condition $x(0)=x_{0}$.

This fact is useful because boundedness is easily established in our examples.

Proposition 3 (Boundedness). Any Krasovskii solution of (BC), (QS), (QB) defined on an interval of the form $[0, T)$ is bounded.

Proof. Let $m$ be any index in $\mathscr{I}$ such that $x_{m}(t)=\min \left\{x_{i}(t), i \in \mathscr{I}\right\}$ and $M$ any index such that $x_{M}(t)=\max \left\{x_{i}(t), i \in \mathscr{I}\right\}$. In the cases of (BC) and (QS), it is straighforward to verify that $x_{m}(t)$ is a non-decreasing function of time and, similarly, that $x_{M}(t)$ is non-increasing. More delicate is the case of (QB), which we verify in detail. Let $q_{m}(t)=q\left(x_{m}(t)\right)$. We have to distinguish three cases. If $x_{m}(t) \in\left(q_{m}(t)-\frac{1}{2}, q_{m}(t)\right]$, then $\dot{x}_{m}(t)=\sum_{j} a_{m j}\left[q\left(x_{j}(t)\right)-x_{m}(t)\right] \geq 0$, because by definition $x_{i}(t) \geq x_{m}(t)$ for $i \in \mathscr{I}$. If $x_{m}(t) \in\left(q_{m}(t), q_{m}(t)+\frac{1}{2}\right)$, then $x_{m}(t)$ may be decreasing as there may be other indices $i$ such that $q\left(x_{i}(t)\right)=q_{m}(t)$. Nevertheless, $\dot{x}_{m}(t) \geq 0$ when $x_{m}(t)=q_{m}(t)$ and then $x_{m}(t)$ remains lower bounded by $\min \left\{x_{m}(0), q_{m}(0)\right\}$ The remaining case when $x_{m}(t)=q_{m}(t)-\frac{1}{2}$ is more delicate and specific to Krasovskii solutions. Indeed, there can exist an index $\ell$ such that $x_{\ell}(t)=x_{m}(t)$ but $q(y)=q_{m}(t)-1$ for some points $y$ in the neighborhood of $x_{\ell}$, which makes the (set-valued) right-hand side include negative values. In such a case, $x_{m}(t)$ would be allowed to decrease, but this fact would in turn lead to the situation of the second case. In conclusion, $x_{m}(t)$ is lower bounded by $q_{m}(0)-1$ and similarly $x_{M}(t)$ is upper bounded by $q_{M}(0)+1$.

Combining the previous two results we readily obtain the following

Corollary 3 (Completeness). Any Carathéodory solution of (QB) or of (BC), as well as any Krasovskii solution of (QB), (QS) or (BC) is defined on $[0,+\infty)$.

This completeness result justifies the analysis of the limit behavior for the dynamics of interest, which we shall undergo in Section 5. Before that, however, we turn our attention to the study of equilibria.

\section{Equilibria: agreement and beyond}

In this section we recall the definitions of equilibria that are natural in our context, we briefly discuss some counterintuitive facts about generalized equilibria, and then we study the equilibria of systems (BC), (QB), and (QS). 
Equilibria are points where a solution can remain indefinitely ${ }^{3}$. In the context of generalized solutions, this general definition leads to distinguish between Carathéodory equilibria and Krasovskii equilibria.

Definition 3 (Equilibria). A point $x^{*}$ is a Carathéodory (Krasovskii) equilibrium of (3) if the function $\varphi(t) \equiv x^{*}, t \geq 0$, is a Carathéodory (Krasovskii) solution of (3).

Carathéodory equilibria are characterized by the equation $f(x)=0$ while Krasovskii equilibria are characterized by the inclusion $0 \in \mathscr{K} f(x)$. Thanks to the multiplicity of solutions, there are examples of non-costant solutions issuing from an equilibrium point.

Example 5 (Escaping from equilibria). Consider the bounded confidence system (BC) with $N=2$ and the initial condition $x_{0}=(-1 / 2,1 / 2)^{\top}$. Let us denote by $f(x)$ the vector field defined by the right-hand side of (BC). Clearly $x_{0}$ is a discontinuity point as $x_{02}-x_{01}-1=0$ and $m=1$ in Assumption 1. Let $s_{1}(x)=x_{2}-x_{1}-1=$ $(-1,1) x-1$. Note that $f\left(x_{0}\right)=0$, then $x_{0}$ is a Carathéodory equilibrium point for the system. On the other hand $f^{(-1)}\left(x_{0}\right)=(1,-1)$ and $\left[(-1,1) f^{(-1)}\left(x_{0}\right)\right](-1)=$ $(-2)(-1)=2>0$. A Carathéodory solution starts from $x_{0}$, enters $S_{(-1)}$ and converges to $(0,0)$.

Being $f(x)$ in (3) allowed to be discontinuous, there may be points which are attractive for Carathéodory solutions without being Carathéodory equilibria: actually, these pathological points are Krasovskii equilibria.

Example 6 (Attractive non-Carathéodory equilibria). Let us consider the quantized behaviour system $(\mathrm{QB})$ over an undirected 4-node path graph with adjacency matrix

$$
A=\left(\begin{array}{llll}
0 & 1 & 0 & 0 \\
1 & 0 & 1 & 0 \\
0 & 1 & 0 & 1 \\
0 & 0 & 1 & 0
\end{array}\right) .
$$

The point $x_{0}=\left(0, \frac{1}{2}, \frac{1}{2}, 1\right)^{\top}$ is attractive for Carathéodory solutions issuing from points in the set $\left.\left\{x \in \mathbb{R}^{4}:-1 / 2 \leq x_{1}, x_{2}<1 / 2,1 / 2 \leq x_{3}, x_{4} \leq 3 / 2\right)\right\}$. Point $x_{0}$ can not be a Carathéodory equilibrium, because $q\left(x_{02}\right)=1 \neq x_{01}=0$, even though it is a Krasovskii equilibrium. A Carathéodory solution originating from $x_{0}$ converges to $(1,1,1,1)^{\top}$.

The following propositions concern equilibria of the systems under consideration. For the dynamics (BC) the sets of Carathéodory and Krasovskii equilibria coincide. The equilibria are precisely those states where individuals either agree or are enough apart not to influence each other, as specified in the following simple result already available in [8].

\footnotetext{
${ }^{3}$ Note that this is a "weak" notion of equilibrium: in case of multiple solutions, we do not require that all solutions remain at the equilibrium.
} 
Proposition 4 (Equilibria of BC). The set of Krasovskii equilibria of (BC) is

$$
\mathscr{F}=\left\{x \in \mathbb{R}^{N}: \text { for every }(i, j) \in \mathscr{I} \times \mathscr{I} \text {, either } x_{i}=x_{j} \text { or }\left|x_{i}-x_{j}\right| \geq 1\right\} \text {. }
$$

In the case of the quantized states dynamics (QS) Carathéodory equilibria and Krasovskii equilibria differ. Carathéodory equilibria are not necessarily consensus points, but the quantizations of their states must agree. The following proposition was proved in [14].

Proposition 5 (Equilibria of QS). The set of Carathéodory equilibria of (QS) is

$$
\mathscr{D}=\left\{x \in \mathbb{R}^{N}: \exists h \in \mathbb{Z} \text { such that } h-\frac{1}{2} \leq x_{i}<h+\frac{1}{2}, \forall i \in \mathscr{I}\right\} .
$$

The set of Krasovskii equilibria of (QS) is $\overline{\mathscr{D}}$.

In case of the quantized behaviours equation (QB) we do not have a characterization of the set of equilibria. On the one hand, we observe that consensus points of the form $h \mathbf{1}$ with $h \in \mathbb{Z}$ are Carathéodory equilibria. On the other hand, there exist equilibria that are far from consensus and are attractive for some Carathéodory solutions. An example is provided in the next result.

Proposition 6 (Far-from-consensus equilibrium of QB). Consider (QB) with an $N$-node path as underlying graph and all non-zero entries of the adjacency matrix A equal to 1 . Then, there exists a Krasovskii equilibrium $x^{*}$ such that

$$
x_{N}^{*}-x_{1}^{*}= \begin{cases}\frac{(N-2)^{2}}{4} & \text { if } N \text { is even } \\ \frac{(N-1)(N-3)}{4} & \text { if } N \text { is odd } .\end{cases}
$$

Proof. The equilibrium can be constructed as follows. We select $\mathbf{k} \in \mathbb{Z}^{N}$ such that $k_{1}=0$ and

$$
k_{i}-k_{i-1}=\left\{\begin{array}{l}
i-2, \quad 2 \leq i \leq \frac{N+2}{2} \\
N-i, \quad \frac{N+2}{2}<i \leq N
\end{array}\right.
$$

and then we set

$$
\begin{aligned}
x_{1}^{*} & =k_{2} \\
x_{i}^{*} & =\frac{k_{i-1}+k_{i+1}}{2}, i=2, \ldots, N-1 \\
x_{N}^{*} & =k_{N-1} .
\end{aligned}
$$

It can be easily verified (details in [17]) that $x^{*}$ is a Krasovskii equilibrium.

\section{Disagreement and distance from consensus}

In the models $(\mathrm{BC}),(\mathrm{QS}),(\mathrm{QB})$ we can not expect to have the same converge properties as (1): in fact they are interesting as they attempt to explain agreement and 
disagreement at the same time. For these models we point out occurrence of disagreement, we give estimates of distance from consensus and, when possible, we give sufficient conditions for convergence to consensus.

\subsection{Bounded confidence dynamics}

In general, the following is the strongest convergence result that has be given about (BC). An example of evolution is in Figure 6.

Theorem 4 (Asymptotic behaviour [15]). Any Krasovskii solution of (BC) converges to a point in $\mathscr{F}$.

In the wake of this fact, much research (from [8] to [49]) has been devoted to understand to which point in $\mathscr{F}$ a solution converges. Since the interaction topology is encoded in the state $x$ by the definition of function $a(\cdot, \cdot)$, conditions should be given in terms of the initial condition $x(0)$. For instance, one can immediately observe that if $\mathscr{G}\left(x_{0}\right)$ is a complete graph, then the dynamics converges to a consensus. More general, though not necessary, conditions for consensus are stated in [51].

Theorem 5 (Sufficient condition for consensus). If $x_{0} \in \mathbb{R}^{N}$ is such that

1. $\mathscr{G}\left(x_{0}\right)$ is connected and

2. for any edge $(i, j) \in \mathscr{E}\left(x_{0}\right)$ the set $\left\{k \in \mathscr{I}:(i, k) \in \mathscr{E}\left(x_{0}\right)\right.$ and $\left.(j, k) \in \mathscr{E}\left(x_{0}\right)\right\}$ has cardinality not smaller than $\frac{N}{2}-2$,

then Krasovskii solutions issuing from $x_{0}$ converge to the consensus point $x_{\text {ave }}(0) \mathbf{1}$.

The previous conditions imply that initial values can not be too much spread. For example, in the case of 10 agents, the distance among agents for $\mathscr{G}\left(x_{0}\right)$ to be connected can be as large as 9 , but in order to satisfy the second condition of Theorem 5 , it can be at most 3 . Other sufficient conditions for consensus may be found by applying the methods in [47].

\subsection{Quantized states dynamics}

Convergence to the set of equilibria can also be proved for dynamics (QS), an example of which is given in Figure 7.

Theorem 6 (Sufficient conditions for discrete consensus [14]). Any Krasovskii solution $\varphi(t)$ of $(\mathrm{QS})$ is such that $\operatorname{dist}(\varphi(t), \mathscr{D}) \rightarrow 0$ as $t \rightarrow+\infty$.

We remark that the set $\mathscr{D}$ is not formed by consensus points, but points in $\mathscr{D}$ are such that $q\left(x_{i}\right)=q\left(x_{j}\right)$ for all $i, j \in \mathscr{I}$. Thus the 2-norm distance of Krasovskii solutions from consensus is, asymptotically, at most $\sqrt{N / 2}$. 


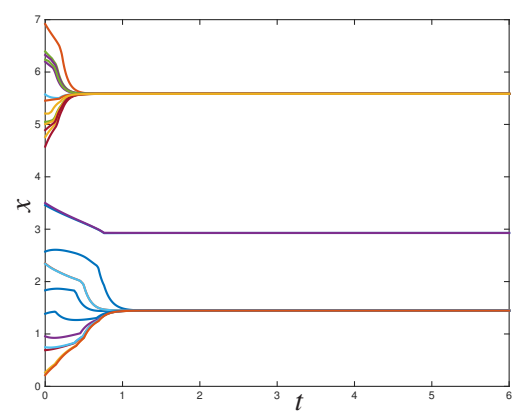

Fig. 6 Evolution of a solution of (BC) from a random initial condition on 25 nodes.

The assumptions that $L$ be symmetric and the interaction graph be undirected can be lifted: recently, [50] has proved the same convergence property for more general functions $q$ and weaker connectivity. Namely, $q$ only needs to be nondecreasing and the graph can be directed and only needs to have a globally reachable node ${ }^{4}$.

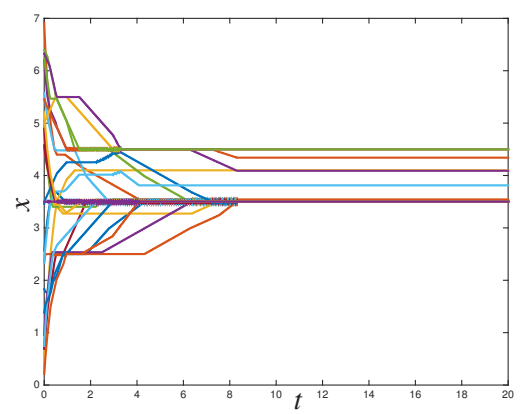

Fig. 7 Evolution of a solution of (QS) on a cycle graph on 25 nodes from the same initial conditions as Figure 6.

\subsection{Quantized behaviour dynamics}

For this system, a general proof of convergence to the equilibria is missing. However, some properties of solutions for large times can be established and are confirmed by simulations, see Figure 7.

\footnotetext{
${ }^{4}$ We refer the reader to [23, Chapter 1] for the relevant definitions about directed graphs.
} 
Theorem 7 (Distance from consensus). If $\varphi(t)$ is any Krasovskii solution of (QB) and

$$
M=\left\{x \in \mathbb{R}^{N}: \inf _{\alpha \in \mathbb{R}}\|x-\alpha \mathbf{1}\| \leq \frac{\|A\|}{\lambda_{*}} \frac{\sqrt{N}}{2}\right\}
$$

then $\operatorname{dist}(\varphi(t), M) \rightarrow 0$ as $t \rightarrow+\infty$.

Proof. First of all we observe that system (QB) can be written

$$
\dot{x}=-L x+A(q(x)-x)
$$

Let $y(t)=x(t)-x_{\text {ave }}(t) \mathbf{1}$. Then $\dot{y}(t)=\dot{x}(t)-\dot{x}_{\text {ave }}(t) \mathbf{1}$. Consider the function $V(y)=$ $\frac{1}{2} y^{\top} y$. We have that

$$
\begin{aligned}
\nabla V(y)^{\top} \dot{y} & =y^{\top} \dot{y} \\
& =\left(x-x_{\text {ave }} \mathbf{1}\right)^{\top}\left[\dot{x}-\dot{x}_{\text {ave }} \mathbf{1}\right] \\
& =\left(x-x_{\text {ave }} \mathbf{1}\right)^{\top} \dot{x}-x^{\top} \dot{x}_{\text {ave }} \mathbf{1}+x_{\text {ave }} \mathbf{1}^{\top} \dot{x}_{\text {ave }} \mathbf{1} \\
& =\left(x-x_{\text {ave }} \mathbf{1}\right)^{\top} \dot{x}-\dot{x}_{\text {ave }} x^{\top} \mathbf{1}+\dot{x}_{\text {ave }} N x_{\text {ave }} \\
& =\left(x-x_{\text {ave }} \mathbf{1}\right)^{\top} \dot{x}-\dot{x}_{\text {ave }} N x_{\text {ave }}+\dot{x}_{\text {ave }} N x_{\text {ave }} \\
& =\left(x-x_{\text {ave }}\right)^{\top} \dot{x}
\end{aligned}
$$

As $L \mathbf{1}=0$, we have

$$
\dot{x} \in-L\left(x-x_{\text {ave }} \mathbf{1}\right)+A \mathscr{K}(q(x)-x) \subseteq-L\left(x-x_{\text {ave }} \mathbf{1}\right)+A(\mathscr{K} q(x)-x) .
$$

For any $v \in \mathscr{K} q(x)-x$, it holds $\|v\| \leq \frac{\sqrt{N}}{2}$. Then, if $v \in \mathscr{K} q(x)-x$ is such that $\dot{y}=-L\left(x-x_{\text {ave }} \mathbf{1}\right)+A v$, we have

$$
\begin{aligned}
\nabla V(y)^{\top} \dot{y} & =\left(x-x_{\text {ave }} \mathbf{1}\right)^{\top}\left[-L\left(x-x_{\text {ave }} \mathbf{1}\right)+A v\right] \\
& =-\left(x-x_{\text {ave }} \mathbf{1}\right)^{\top} L\left(x-x_{\text {ave }} \mathbf{1}\right)+\left(x-x_{\text {ave }} \mathbf{1}\right)^{\top} A v \\
& \leq-\lambda_{*}\left\|x-x_{\text {ave }} \mathbf{1}\right\|^{2}+\left\|x-x_{\text {ave }} \mathbf{1}\right\|\|A\| \frac{\sqrt{N}}{2} \\
& \leq\left\|x-x_{\text {ave }} \mathbf{1}\right\|\left[-\lambda_{*}\left\|x-x_{\text {ave }} \mathbf{1}\right\|+\|A\| \frac{\sqrt{N}}{2}\right] .
\end{aligned}
$$

We conclude that $\operatorname{dist}(x(t), M) \rightarrow 0$ as $t \rightarrow+\infty$, because otherwise $V$ would decrease unboundedly along solutions, which is forbidden by $V$ being nonnegative.

We remark that this result is tight in the following sense: on some graphs, the estimate on the limit set is asymptotically tight for large networks in the sense of the Euclidean distance from the consensus. More precisely, if the graph is a path with $N$ nodes and weights are uniform, for all points in the attractor $M$ it holds true that $\frac{1}{\sqrt{N}}\left\|x-x_{\text {ave }}\right\|=O\left(N^{2}\right)$ as $N \rightarrow \infty$. At the same time, the equilibrium $x^{*}$ that was constructed in the proof of Proposition 6 is such that (for odd $N$ ) 


$$
\frac{1}{\sqrt{N}}\left\|x^{*}-x_{\text {ave }}^{*}\right\|=\frac{1}{\sqrt{120}} N^{2}+o\left(N^{2}\right) \quad \text { as } N \rightarrow \infty .
$$

Hence, the estimate of $M$ can not be improved in general in terms of distance from consensus. Details of these computations can be found in [17].

Even though not guaranteed in general, consensus is achieved on some topologies. An example of such result is the following.

Theorem 8 (Sufficient conditions for consensus). If the graph underlying system (QB) is either complete or complete bipartite and its adjacency matrix A has all non-null entries equal to 1 , then all Krasovskii solutions of (QB) converge to a consensus point.

The proof of this result, which can be found in [17], is based on showing that $\max _{i} x_{i}(t)-\min _{i} x_{i}(t)$ is decreasing and converges to zero.

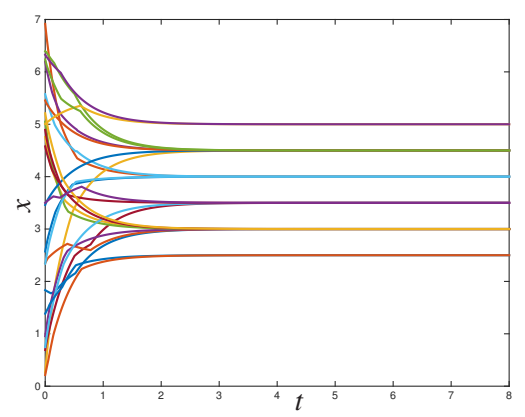

Fig. 8 Evolution of a solution of (QB), assuming the same initial conditions and graph as Figure 7.

\section{Discussion: the origins of disagreement in opinion dynamics}

The dynamics analysed in this paper are meant to describe opinion dynamics in social networks. In this context, the nodes of the graph are individuals, an edge between two nodes means that they socially interact and the $i$-th component of the state represents the value of the $i$-th individual's opinion. This graph-based modeling approach has a strong support in mathematical sociology [27, 43] as well as in economics [36] and in the physics of complex systems [11,32,5].

The basic assumption in these models of opinion dynamics is that if an individual communicates with another, then his/her opinion is attracted by the other's. If one translates this assumption into a set of differential equations, then one gets system (1), as already proposed in [1]. This dynamics asymptotically leads to consensus, i.e. agreement of the individuals on the same opinion, except in case there are 
different groups of individuals which do not communicate with each other, i.e. the communication graph has separated connected components. However, it has been noted that agreement is rare in societies [28], even if individuals do communicate: for this reason, more complex models have been elaborated with the aim of explaining agreement and disagreement at the same time.

In this paper we have focused on a group of models involving different kinds of threshold phenomena leading to discontinuities. Before going back to discuss their features, it is important to mention that these are not the only possible explanations for disagreement. In [29] disagreement is explained as the effect of obstinacy, that is translated into the dependence of any individual's opinion on its initial value. Stubborness as the source of disagreement is also considered in other models, such as $[39,42]$, also in connection with the occurrence of randomized asynchronous interactions $[2,25,45]$. Another explanation has been proposed to be the presence of contrarians [31] or of negative interactions, i.e. negative weights in the adjacency matrix [3]. Similar dynamics on "signed graphs" may also feature randomized interactions [46] or bounded confidence [18].

Going back to the models considered in this paper, we now try to summarize their features in the opinion dynamics context, beginning with (BC). Bounded Confidence dynamics allow for the existence of complete Carathéodory solutions for every initial condition and all Krasovskii solutions are proved to converge to an equilibrium. The structure of these equilibria is a set of separated clusters of individuals sharing the same opinion. In $[8,15]$ it is proved that, due to robustness issues, one can expect the opinion values of different clusters to be approximately twice the threshold apart. The most recent results on this matter are probably those in [49]. Actually, a fine understanding of how the final opinions depend on the initial ones is still missing. In this chapter, we have reported a sufficient condition for consensus, which asks for the initial opinions to be already quite close to each other. Other models that involve assumptions of bounded confidence include [22, 38, 9, 52, 26].

Consensus dynamics with quantization have first been studied with engineering motivations, while seeking controlled dynamics that could lead to (approximate) consensus despite the constraint of quantization [10, 41]. Proposed in this context by [14], the quantized states dynamics (QS) does not allow for global existence of Carathéodory solutions and thus requires to consider Krasovskii solutions: all Krasovskii solutions converge to equilibria such that the quantized opinions are equal. This is not exactly consensus, as individuals' opinions may slightly differ, but they agree on their quantized values. Consistently with its history, dynamics (QS) better fits engineering applications than social dynamics ${ }^{5}$ : we believe that a better model of quantized social interactions is given by the quantized behavior dynamics (QB), which we proposed in [16, 17]. This model allows for the existence of complete Carathéodory solutions for every initial conditions, but Krasovskii solutions are preferred to avoid the pathology of solutions converging to non-equilibrium point. In general, a result of convergence to equilibria is missing, but a tight result of convergence to a set is available. Remarkably, there can be equilibria very far

\footnotetext{
5 The discretization of the opinions in social systems has been observed by social scientists [30,
} Chapter 10] and addressed in several models including [48, 37, 20]. 
from consensus, in which the difference among different opinions of individuals is proportional to $N^{2}$.

Beyond the specific dynamics considered in this chapter, we believe that dynamical models that involve discontinuities can be useful in the study of social dynamics: we thus hope that the tools collected here can also be useful in the analysis of new and richer models.

\section{References}

1. Abelson, R.P.: Mathematical models in social psychology. Advances in experimental social psychology 3, 1-54 (1967)

2. Acemoğlu, D., Como, G., Fagnani, F., Ozdaglar, A.: Opinion fluctuations and disagreement in social networks. Mathematics of Operations Research 38(1), 1-27 (2013)

3. Altafini, C.: Consensus problems on networks with antagonistic interactions. IEEE Transactions on Automatic Control 58(4), 935-946 (2013)

4. Aubin, J.P., Cellina, A.: Differential inclusions, Grundlehren der Mathematischen Wissenschaften, vol. 264. Springer, Berlin (1984)

5. Aydoğdu, A., Caponigro, M., McQuade, S., Piccoli, B., Pouradier Duteil, N., Rossi, F., Trélat, E.: Interaction Network, State Space, and Control in Social Dynamics, Springer International Publishing (2017) DOI: 10.1007/978-3-319-49996-3_3

6. Bacciotti, A.: Some remarks on generalized solutions of discontinuous differential equations. International Journal of Pure and Applied Mathematics 10(3), 257-266 (2003)

7. Blondel, V.D., Hendrickx, J.M., Tsitsiklis, J.N.: Existence and uniqueness of solutions for a continuous-time opinion dynamics model with state-dependent connectivity (2009). URL http://www.mit.edu/jnt/Papers/BHT10-solutions-DA.pdf

8. Blondel, V.D., Hendrickx, J.M., Tsitsiklis, J.N.: Continuous-time average-preserving opinion dynamics with opinion-dependent communications. SIAM Journal on Control and Optimization 48(8), 5214-5240 (2010)

9. Canuto, C., Fagnani, F., Tilli, P.: An Eulerian approach to the analysis of Krause's consensus models. SIAM Journal on Control and Optimization 50(1), 243-265 (2012)

10. Carli, R., Fagnani, F., Speranzon, A., Zampieri, S.: Communication constraints in the average consensus problem. Automatica 44(3), 671-684 (2008)

11. Castellano, C., Fortunato, S., Loreto, V.: Statistical physics of social dynamics. Reviews of Modern Physics 81(2), 591-646 (2009)

12. Ceragioli, F.: Discontinuous ordinary differential equations and stabilization. Ph.D. thesis, Università di Firenze (2000)

13. Ceragioli, F.: Finite valued feedback laws and piecewise classical solutions. Nonlinear Analysis: Theory, Methods \& Applications 65(5), 984-998 (2006)

14. Ceragioli, F., De Persis, C., Frasca, P.: Discontinuities and hysteresis in quantized average consensus. Automatica 47(9), 1916-1928 (2011)

15. Ceragioli, F., Frasca, P.: Continuous and discontinuous opinion dynamics with bounded confidence. Nonlinear Analysis: Real World Applications 13(3), 1239-1251 (2012)

16. Ceragioli, F., Frasca, P.: Continuous-time consensus dynamics with quantized all-to-all communication. In: European Control Conference, pp. 1120-1125. Linz, Austria (2015)

17. Ceragioli, F., Frasca, P.: Consensus and disagreement: the role of quantized behaviours in opinion dynamics (2016). URL http://arxiv.org/abs/1607.01482. Submitted

18. Ceragioli, F., Lindmark, G., Veibäck, C., Wahlström, N., Lindfors, M., Altafini, C.: A bounded confidence model that preserves the signs of the opinions. In: European Control Conference 2016, pp. 543-548. Aalborg, Denmark (2016)

19. Chazelle, B., Wang, C.: Inertial Hegselmann-Krause systems. IEEE Transactions on Automatic Control 62(8), 3905-3913 (2016) 
20. Chowdhury, N.R., Morarescu, I.C., Martin, S., Srikant, S.: Continuous opinions and discrete actions in social networks: a multi-agent system approach. In: IEEE Conference on Decision and Control, pp. 1739-1744. Las Vegas, NV, USA (2016)

21. Cortés, J.: Discontinuous dynamical systems - a tutorial on solutions, nonsmooth analysis, and stability. IEEE Control Systems Magazine 28(3), 36-73 (2008)

22. Deffuant, G., Neau, D., Amblard, F., Weisbuch, G.: Mixing beliefs among interacting agents. Advances in Complex Systems 3(1-4), 87-98 (2000)

23. Fagnani, F., Frasca, P.: Introduction to Averaging Dynamics over Networks. Lecture Notes in Control and Information Sciences. Springer (2017)

24. Filippov, A.: Differential Equations with Discontinuous Righthandside. Kluwer, Berlin (1988)

25. Frasca, P., Ravazzi, C., Tempo, R., Ishii, H.: Gossips and prejudices: Ergodic randomized dynamics in social networks. In: IFAC Workshop on Estimation and Control of Networked Systems, pp. 212-219. Koblenz, Germany (2013)

26. Frasca, P., Tarbouriech, S., Zaccarian, L.: A hybrid model of opinion dynamics with limited confidence. In: IFAC Symposium on Nonlinear Control Systems. Monterey, CA, USA (2016)

27. Friedkin, N.E.: A structural theory of social influence. Cambridge University Press (2006)

28. Friedkin, N.E.: The problem of social control and coordination of complex systems in sociology: A look at the community cleavage problem. IEEE Control Systems 35(3), 40-51 (2015)

29. Friedkin, N.E., Johnsen, E.C.: Social influence networks and opinion change. In: E.J. Lawler, M.W. Macy (eds.) Advances in Group Processes, vol. 16, pp. 1-29. JAI Press (1999)

30. Friedkin, N.E., Johnsen, E.C.: Social Influence Network Theory: A Sociological Examination of Small Group Dynamics. Cambridge University Press (2011)

31. Galam, S.: Contrarian deterministic effects on opinion dynamics: "the hung elections scenario". Physica A: Statistical Mechanics and its Applications 333(Supplement C), 453-460 (2004)

32. Galam, S.: Sociophysics: a physicist's modeling of psycho-political phenomena. Springer Science \& Business Media (2012)

33. Hájek, O.: Discontinuous differential equations I. Journal of Differential Equations 32, 149170 (1979)

34. Hegselmann, R., Krause, U.: Opinion dynamics and bounded confidence: models, analysis and simulation. Journal of Artificial Societies and Social Simulation 5(3), 1-33 (2002)

35. Jabin, P.E., Motsch, S.: Clustering and asymptotic behavior in opinion formation. Journal of Differential Equations 257(11), 4165 - 4187 (2014)

36. Jackson, M.O.: Social and economic networks. Princeton University Press (2010)

37. Martins, A.C.R.: Continuous opinions and discrete actions in opinion dynamics problems. International Journal of Modern Physics C 19(04), 617-624 (2008)

38. Mirtabatabaei, A., Bullo, F.: Opinion dynamics in heterogeneous networks: Convergence conjectures and theorems. SIAM Journal on Control and Optimization 50(5), 2763-2785 (2012)

39. Mobilia, M.: Does a single zealot affect an infinite group of voters? Physical Review Letters 91(2), 028,701 (2003)

40. Motsch, S., Tadmor, E.: Heterophilious dynamics enhances consensus. SIAM Review 56(4), 577-621 (2014)

41. Nedic, A., Olshevsky, A., Ozdaglar, A., Tsitsiklis, J.N.: On distributed averaging algorithms and quantization effects. IEEE Transactions on Automatic Control 54(11), 2506-2517 (2009)

42. Parsegov, S.E., Proskurnikov, A.V., Tempo, R., Friedkin, N.E.: Novel multidimensional models of opinion dynamics in social networks. IEEE Transactions on Automatic Control 62(5), 2270-2285 (2017)

43. Proskurnikov, A.V., Tempo, R.: A tutorial on modeling and analysis of dynamic social networks. Part I. Annual Reviews in Control 43, 65-79 (2017)

44. Pucci, A.: Traiettorie di campi di vettori discontinui. Rendiconti dell'Istituto Matematico dell'Università degli Studi di Trieste 8, 84-93 (1976)

45. Ravazzi, C., Frasca, P., Tempo, R., Ishii, H.: Ergodic randomized algorithms and dynamics over networks. IEEE Transactions on Control of Network Systems 2(1), 78-87 (2015) 
46. Shi, G., Proutiere, A., Johansson, M., Baras, J.S., Johansson, K.H.: The evolution of beliefs over signed social networks. Operations Research 64(3), 585-604 (2016)

47. Tangredi, D., Iervolino, R., Vasca, F.: Consensus stability in the Hegselmann-Krause model with coopetition and cooperosity. In: IFAC World Congress, pp. 12,426-12,431. Toulouse, France (2017)

48. Urbig, D.: Attitude dynamics with limited verbalisation capabilities. Journal of Artificial Societies and Social Simulation 6(1), 1-23 (2003)

49. Wang, C., Li, Q., Weinan, E., Chazelle, B.: Noisy Hegselmann-Krause systems: Phase transition and the 2r-conjecture. Journal of Statistical Physics 166(5), 1209-1225 (2017)

50. Wei, J., Yi, X., Sandberg, H., Johansson, K.: Nonlinear consensus protocols with applications to quantized systems. In: IFAC World Congress, pp. 16,010-16,015. Toulouse, France (2017)

51. Yang, Y., Dimarogonas, D.V., Hu, X.: Opinion consensus of modified Hegselmann-Krause models. Automatica 50(2), 622-627 (2014)

52. Zhang, J., Hong, Y.: Opinion evolution analysis for short-range and long-range DeffuantWeisbuch models. Physica A 392(21), 5289-5297 (2013) 